\title{
Overlap Between Genetic Variants Associated With Schizophrenia and Intelligence Quotient: Protocol for a Systematic Review
}

Nancy Murillo-García ( $\sim$ psi.nmurillo@gmail.com )

Fundacion Marques de Valdecilla https://orcid.org/0000-0002-7337-8309

Sara Barrio-Martínez

Valdecilla Biomedical Research Institute (IDIVAL)

Esther Setién-Suero

Valdecilla Biomedical Research Institute (IDIVAL)

Rosa Ayesa-Arriola

Valdecilla Biomedical Research Institute (IDIVAL)

\section{Protocol}

Keywords: schizophrenia, intelligence quotient, genetic variants, polymorphism

Posted Date: January 20th, 2021

DOI: https://doi.org/10.21203/rs.3.rs-150210/v1

License: (a) (i) This work is licensed under a Creative Commons Attribution 4.0 International License. Read Full License 


\section{Abstract}

\section{Background}

People with schizophrenia often exhibit a premorbid intelligence quotient (IQ) deficit. A genetic overlap between schizophrenia and IQ has been found, indicating shared genetic variants underlying the risk of schizophrenia and a low IQ. However, literature has emerged that offers contradictory findings about this issue. The aim of this systematic review will be to analyse and summarize the evidence that explores genetic variants associated with schizophrenia and IQ.

Methods

A search will be carried out in four electronic databases (MEDLINE via PubMed, PsycINFO, Web of Science and Scopus). The search strategy will include terms related to "schizophrenia", "genetic variants" and "intelligence quotient", which will be adapted to medical subject headings (Mesh) format, Thesaurus format and free text. The team of reviewers will record the results in a bibliographic manager software and conduct the selection process. Observational studies examining human adults with schizophrenia that explore the association between genetic variants and the IQ of the participants will be considered. The risk of bias of the primary studies will be assessed using the genetic study quality tool (Q-Genie). After data extraction, the main results will be presented in a narrative synthesis.

\section{Discussion}

The findings of this systematic review may help us understand if there is genetic overlap between schizophrenia and IQ, and to identify specific genetic factors involved.

Systematic review registration

PROSPERO CRD42020218842.

\section{Background}

Schizophrenia is a chronic mental disorder characterized by a distinctive symptomatology that includes hallucinations, delusions, disorganized thinking and functional impairment $(1,2)$. There is great variability in its epidemiology, multiple clinical presentations and generally begins in late adolescence or early adulthood (3). Schizophrenia ranked the 12th most disabling disorder among 310 diseases and injuries globally in 2016 (4), affecting around 21 million people worldwide (5).

Cognitive deficit is a central feature in schizophrenia, and is often present before the onset of the disease. These findings suggest that cognitive dysfunction could be a risk factor for developing schizophrenia (68). In this line, a lower intelligence quotient (IQ) has been observed in children who later developed schizophrenia (9), in individuals at high risk of suffering it (10), and in relatives of patients with the disorder (11). The meta-analysis by Khandaker et al., where different cohort studies were included, 
confirmed a premorbid IQ deficit in people with schizophrenia, who showed a mean IQ of 93.6 (6). Moreover, Kendler et al. found that deviation of the individual IQ from the family IQ conferred risk of developing schizophrenia (12). Therefore, when people do not reach their parents intelligence they would have an increased susceptibility for the disease. Given that the IQ is hereditary (13), reliable and stable (14), it has been proposed as a promising endophenotype of the disease.

In addition to the efforts for recognizing observable endophenotypes of schizophrenia, there is also interest in identifying genetic markers of the disease. It has been proven that schizophrenia has a strong genetic base with an estimated heritability of about $80 \%(6,7)$. However, this disorder is genetically and phenotypically complex, as it is influenced by both genomic and environmental factors that interact regulating genetic expression (8). To date, genome-wide association studies (GWAS) evidenced that a substantial proportion of the heritability of schizophrenia is explained by a polygenic effect involving thousands of single nucleotide polymorphisms (SNPs) of small effect. In a large-scale GWAS, the Schizophrenia Working Group of the Psychiatric Genomics Consortium (PGC) identified 108 loci related to the disorder (9). More recently, a meta-analysis found another 50 loci associated to schizophrenia (10).

Interestingly, an overlap between genetic factors associated with vulnerability to schizophrenia and neurocognitive function has been found. The Cognitive Genomics Consortium (COGENT) reported that polygenic risk score (PRS) for schizophrenia is related to lower general cognitive ability (11). In the general population, Sniekers et al. obtained a genetic correlation between IQ and schizophrenia (12). In addition, SNPs such as rs1011313, located in genes involved in the glutamatergic system (DTNBP1) have been related to both neurocognition and susceptibility to schizophrenia (13). These data indicate possible shared genetic factors predisposing to schizophrenia and low IQ, so their identification would contribute to understand the biological mechanisms of the disorder.

Previous bibliographic reviews addressing the relationship between these variables lack a systematic methodology or a description of the search strategies $(14,15)$. Furthermore, there is currently no bibliographic review that focuses specifically on the genetic variants associated with both phenotypes. The objective of this systematic review will be to to analyze the existing literature on genetic variants associated with both schizophrenia and IQ to identify possible polymorphisms underlying the genetic overlap.

\section{Methods And Analysis}

For the elaboration of this protocol, the Preferred Reporting Items for Systematic Reviews and MetaAnalysis Protocols (PRISMA-P) guideline was followed (see the checklist in Additional file 1). This study has been registered in the International Prospective Registry of Systematic Reviews (PROSPERO CRD42020218842).

\section{Eligibility criteria}


Studies that meet the following criteria will be selected: a) studies examining human adult population with diagnosis of a schizophrenia spectrum disorder; b) genetic studies exploring genetic variants including single nucleotide polymorphisms (SNPs), copy number variations (CNV), genomic insertions or genomic deletions; $\mathrm{C}$ ) studies estimating the participants premorbid IQ and exploring its association with genetic variants; d) original studies with an observational design, including cross-sectional, case-control and cohort studies; e) studies written in English.

Studies with the following characteristics will be excluded: a) animal model or cell line studies; b) studies that lack the genotyping or IQ estimation of the participants, as well as studies that do not address the relationship between these variables; c) studies that use PRS that do not identify specific genetic variants; d) review or meta-analysis articles; d) not peer-reviewed literature, single case studies, books, editorials or theses.

\section{Outcomes}

This systematic review will analyze the overlap between the genetic variants associated with both schizophrenia and IQ. Therefore, the main outcome will be evidence of genetic association between these two phenotypes, found through GWAS or candidate gene strategies. Specific genetic variants or genes that are associated with the disease and the IQ will be described.

If studies comparing individuals with schizophrenia and healthy controls are found, these results will be analyzed as additional outcomes. The IQ of the participants will also be compared between different studies, and possible differences between men and women will be explored.

\section{Search strategies and information source}

The bibliographic search will be carried out in the electronic databases of MEDLINE via PubMed, PsycINFO, Web of Science (WOS) and Scopus. For the search strategy, Mesh terms related to "schizophrenia", "genetic variants" and "intelligence quotient" will be used to explore MEDLINE. Similarly, these terms will be adapted to the Thesaurus format for searching PsycINFO. Subsequently, this strategy will be adjusted to free text in order to search the other databases (see Additional file 2). To narrow the results, the document type (article) will be set as limit. The strategy described was established after consulting an expert librarian in Health Sciences from the University of Cantabria (Santander, Spain).

\section{Study records and selection process}

Once the electronic databases have been searched, all the results will be recorded in EndNote, a bibliographic manager. Subsequently, the duplicate records will be eliminated and the team of reviewers (NMG, SBM) will evaluate the titles and abstracts of each article, identifying candidates for inclusion. In the next phase, the team of reviewers will analyze the full text of these articles independently, selecting those that meet the eligibility criteria described above. Doubts and discrepancies will be consulted with a postdoctoral researcher (ESS) and a senior researcher (RAA), and will be solved in consensus by the entire team. The article selection process will be recorded and presented in a PRISMA flow chart. 


\section{Assessment of risk of bias of primary studies}

The literature quality evaluation will be independently performed by two reviewers using the quality of genetic studies (Q-Genie) tool, which evaluate the risk of bias of genetic association studies (16). This tool contains 11 items that are rated on a 7-point Likert scale ( $1=$ poor quality, $7=$ excellent quality) to assess different categories. A global score can be estimated, indicating if the overall quality of the study is poor (scores $\leq 35$ for studies with control groups and scores $\leq 32$ for studies without control groups), moderate (total scores $>35$ and $\leq 45$ for studies with control groups and scores $>32$ and $\leq 40$ for studies without control groups) or good (total scores $>45$ for studies with control groups and scores $>40$ for studies without control groups). The discrepancies will be solved by consensus with the entire review team, and the global scores of the studies will be presented in the results section.

\section{Data extraction}

The data will be extracted by the team of reviewers. First, titles and abstracts will be screened for potential inclusion in the systematic review. Those articles that meet the inclusion criteria will be selected and the full text will be reviewed. A standardized table will be designed by the entire team, where all data will be recorded, including: author, year of publication, country of origin, study design, type of genetic study, ethnicity, genetic variants investigated, genes investigated, sample size (patients and healthy controls if applicable), percentage of men and women, mean age, mean IQ, instruments for IQ measuring and main findings.

\section{Data synthesis}

A narrative synthesis of all the relevant data of the studies included in the review will be presented, including a summary table adapted from the Excel table where the data extraction was carried out. The data will be grouped in the results section according to the type of genetic variation and the gene investigated in the different studies. It will be considered the genetic overlap between both phenotypes.

\section{Discussion}

Here, we presented a protocol for conducting a systematic review on genetic variants associated with schizophrenia and intelligence quotient (IQ) to identify potential overlaps. Both phenotypes have shown to be hereditary and related to genetic variation, mostly explained by thousands of SNPs with small effect. In addition, a set of genetic factors linked to risk of schizophrenia and low general cognitive ability have been observed (11). However, other studies found no evidence of genetic overlapping between the two $(17,18)$. Therefore, a summary of current literature on this relationship is justified to advance knowledge of the biological mechanisms of schizophrenia and the identification of endophenotypes useful for its diagnosis.

Confirming that genetic variants associated with the disease are also involved in IQ would suggest that genetic factors that confer risk of schizophrenia also affect neurocognition. Evidence of shared genetic variation between these two phenotypes would support the neurodevelopmental hypothesis, which 
proposes that the etiology of schizophrenia is related to pathological brain development $(19,20)$. Therefore, the identification of a genetic base predisposing to schizophrenia and lower premorbid IQ would indicate a molecular pathway towards the disease since early stages in life. Moreover, a genetic overlap for risk of schizophrenia and low premorbid IQ would show that IQ is a valid endophenotype for the disorder (21). In consequence, the IQ estimation could contribute to detect individuals with risk of developing schizophrenia, and to help the diagnosis. Otherwise, if a small shared genetic variance is found between schizophrenia and IQ, or if a lack of association between its genetic factors is evidenced; this would indicate that the neurocognitive deficits observed in patients with schizophrenia may be secondary to the disorder or another environmental variables (18).

The results of this systematic review will serve as a basis for directing future research on the pathogenesis of psychotic disorders. Lately, the research about schizophrenia and intelligence has generated wide interest in researchers. However, most of the currently available reviews and metaanalysis have focused on the study of the correlation between these two phenotypes, regardless of their genetic variants (22-24). There is little literature which considers the genetic overlap of both phenotypes. In the review by Ohi et al. (15), common genetic variants were found between schizophrenia and cognitive functioning. Interestingly, it takes into account different measures related to general cognitive function, but does not focus on IQ specifically. The same is true in the review by Smeland \& Andreassen (25), where a genetic overlap between schizophrenia and cognitive dysfunction is shown. In addition, neither investigation reported on the methodology used. Also, there is a meta-analysis that considers the different genetic variants of intelligence (12). However, this study takes into account multiple disorders and diseases, without specifically focusing on schizophrenia. It is our hope that we can solve these constraints.

As far as we know, this will be the first systematic review that studies the genetic overlap between schizophrenia and IQ, integrating all these shared variants into one document. However, we are aware of the existence of certain limitations in this review. Mainly, we refer to the great heterogeneity expected in terms of the different genotyping techniques, as well as different tools used when evaluating IQ.

\section{Abbreviations}

Cognitive Genomics Consortium (COGENT), Copy Number Variations (CNV), Dystrobrevin binding protein 1 (DTNBP1), Genetic study quality tool (Q-Genie), Genome-wide association studies (GWAS), Intelligence quotient (IQ), Medical subject headings (Mesh), Polygenic risk score (PRS), Preferred Reporting Items for Systematic Reviews And Meta-Analysis Protocols (PRISMA-P), Schizophrenia Working Group of the Psychiatric Genomics Consortium (PGC), Single nucleotide polymorphisms (SNPs), International Prospective Registry of Systematic Reviews (PROSPERO),Web of Science (WOS).

\section{Declarations}

\section{Ethics approval and consent to participate}


Not applicable.

\section{Consent for publication}

Not applicable.

\section{Availability of data and materials}

All data generated or analysed during this study are included in this published article [and its supplementary information files].

\section{Competing interests}

The authors declare that they have no competing interests.

\section{Funding}

This work was supported by a "Miguel Servet" contract (Dr. Rosa Ayesa-Arriola) from the Carlos III Health Institute (CP18/00003); and a predoctoral contract (Nancy Murillo-Garcia) from the Valdecilla Biomedical Research Institute and the University of Cantabria (BOC49, REF. IDI-13).

\section{Authors' contributions}

NMG and RAA envisioned and planned the study.

ESS and RAA guided and supervised the design of the methods for the systematic review.

NMG and SBM designed the search strategy and elaborated the initial draft of this protocol.

RAA revised the protocol.

All authors read and approved the final version of this protocol.

\section{Acknowledgements}

Not applicable.

\section{References}

1. Association AP. Diagnostic and Statistical Manual of Mental Disorders (DSM-IV). 4th ed. Washington DC: American Psychological Association; 1994.

2. Saha S, Chant D, Welham J, McGrath J. A systematic review of the prevalence of schizophrenia. PLoS Med. 2005;2(5):31.

3. Schultz SK, Andreasen NC. Schizophrenia. Lancet. 1999;353(9162):1425-30. 
4. Collaborators GDallaP. Global, regional, and national incidence, prevalence, and years lived with disability for 328 diseases and injuries for 195 countries, 1990-2016: a systematic analysis for the Global Burden of Disease Study 2016. Lancet. 2017;390(10100):1211-59.

5. Collaborators GDallaP. Global, regional, and national incidence, prevalence, and years lived with disability for 354 diseases and injuries for 195 countries and territories, 1990-2017: a systematic analysis for the Global Burden of Disease Study 2017. Lancet. 2018;392(10159):1789-858.

6. Lichtenstein P, Yip BH, Björk C, Pawitan Y, Cannon TD, Sullivan PF, et al. Common genetic determinants of schizophrenia and bipolar disorder in Swedish families: a population-based study. Lancet. 2009 Jan 17;373(9659):234-9.

7. Sullivan PF, Kendler KS, Neale MC. Schizophrenia as a complex trait: evidence from a meta-analysis of twin studies. Arch Gen Psychiatry. 2003 Dec;60(12):1187-92.

8. van de Leemput J, Hess JL, Glatt SJ, Tsuang MT. Genetics of Schizophrenia: Historical Insights and Prevailing Evidence. Adv Genet. 2016;96:99-141.

9. Ripke S, Neale BM, Corvin A, Walters JTR, Farh K-H, Holmans PA, et al. Biological insights from 108 schizophrenia-associated genetic loci. Nature. 2014 2014/07/01;511(7510):421-7.

10. Pardiñas AF, Holmans P, Pocklington AJ, Escott-Price V, Ripke S, Carrera N, et al. Common schizophrenia alleles are enriched in mutation-intolerant genes and in regions under strong background selection. Nature Genetics. 2018 2018/03/01;50(3):381-9.

11. Lencz T, Knowles E, Davies G, Guha S, Liewald DC, Starr JM, et al. Molecular genetic evidence for overlap between general cognitive ability and risk for schizophrenia: a report from the Cognitive Genomics consorTium (COGENT). Mol Psychiatry. 2014 Feb;19(2):168-74.

12. Sniekers S, Stringer S, Watanabe K, Jansen PR, Coleman JRI, KrapohI E, et al. Genome-wide association meta-analysis of 78,308 individuals identifies new loci and genes influencing human intelligence. Nature Genetics. 2017 2017/07/01;49(7):1107-12.

13. Yang Y, Zhang L, Guo D, Yu H, Liu Q, Su X, et al. Association of DTNBP1 With Schizophrenia: Findings From Two Independent Samples of Han Chinese Population. Front Psychiatry. 2020;11:446.

14. Burdick KE, Gunawardane N, Woodberry K, Malhotra AK. The role of general intelligence as an intermediate phenotype for neuropsychiatric disorders. Cogn Neuropsychiatry. 2009;14(4-5):299-311.

15. Ohi K, Sumiyoshi C, Fujino H, Yasuda Y, Yamamori H, Fujimoto M, et al. Genetic Overlap between General Cognitive Function and Schizophrenia: A Review of Cognitive GWASs. Int J Mol Sci. 2018 Nov 30;19(12).

16. Sohani ZN, Meyre D, de Souza RJ, Joseph PG, Gandhi M, Dennis BB, et al. Assessing the quality of published genetic association studies in meta-analyses: the quality of genetic studies (Q-Genie) tool. BMC Genet. 2015 May 15;16:50.

17. Fowler T, Zammit S, Owen MJ, Rasmussen F. A population-based study of shared genetic variation between premorbid IQ and psychosis among male twin pairs and sibling pairs from Sweden. Arch Gen Psychiatry. 2012 May;69(5):460-6. 
18. van Scheltinga AF, Bakker SC, van Haren NE, Derks EM, Buizer-Voskamp JE, Cahn W, et al. Schizophrenia genetic variants are not associated with intelligence. Psychol Med. 2013 Dec;43(12):2563-70.

19. Owen MJ, O'Donovan MC. Schizophrenia and the neurodevelopmental continuum:evidence from genomics. World Psychiatry. 2017 Oct;16(3):227-35.

20. Rund BR. The research evidence for schizophrenia as a neurodevelopmental disorder. Scand $J$ Psychol. 2018 Feb;59(1):49-58.

21. Greenwood TA, Lazzeroni LC, Maihofer AX, Swerdlow NR, Calkins ME, Freedman R, et al. Genomewide Association of Endophenotypes for Schizophrenia From the Consortium on the Genetics of Schizophrenia (COGS) Study. JAMA Psychiatry. 2019 Oct 9;76(12):1274-84.

22. Aylward E, Walker E, Bettes B. Intelligence in schizophrenia: meta-analysis of the research. Schizophr Bull. 1984;10(3):430-59.

23. Khandaker GM, Barnett JH, White IR, Jones PB. A quantitative meta-analysis of population-based studies of premorbid intelligence and schizophrenia. Schizophr Res. 2011 Nov;132(2-3):220-7.

24. Kristen A. Woodberry MSW, A.M. „Anthony J. Giuliano PD, Larry J. Seidman PD. Premorbid IQ in Schizophrenia: A Meta-Analytic Review. American Journal of Psychiatry. 2008;165(5):579-87.

25. Smeland OB, Wang Y, Frei O, Li W, Hibar DP, Franke B, et al. Genetic Overlap Between Schizophrenia and Volumes of Hippocampus, Putamen, and Intracranial Volume Indicates Shared Molecular Genetic Mechanisms. Schizophr Bull. 2018 Jun 6;44(4):854-64.

\section{Supplementary Files}

This is a list of supplementary files associated with this preprint. Click to download.

- Additionalfile1.doc

- Additionalfile2.doc 